\title{
Hybrid Velocity/Force Control for Robot Navigation in Compliant Unknown Environments
}

\author{
Dushyant Palejiya and Herbert G. Tanner
}

\begin{abstract}
We combine a "hybrid" force/position control scheme with a potential field approach into a novel method for collision recovery and navigation in unknown environments. It can be implemented both on manipulators and mobile robots. The use of force sensors allows us to locally sense the environment and design a dynamic control law. Multiple Lyapunov functions are used to establish asymptotic stability of the closed loop system. The switching conditions and stability criteria are established under reasonable assumptions on the type of obstacles present in the environment. Extensive simulation results are presented to illustrate the system behavior under designed control scheme, and verify its stability, collision recovery and navigation properties.
\end{abstract}




\section{Introduction}

\subsection{Motivation and Overview}

We address the problem of steering a redundant manipulator or a mobile robot inside partially unknown environment. The environment is assumed to contain compliant objects and the robot is required to use only its force/torque sensors in order to navigate. Motivation for this approach comes primarily from the need for coping with uncertainty in robotic search and rescue operations. Contrary to the case of industrial environments, where the environment can be structured and regulated, in calamity situations uncertainty prevails. when a robotic snake or a tethered mobile robot dives into pile of debris, there is little hope of getting GPS signals, getting enough light for image processing, or using ultrasound sensors in such confined environments. In such cases, most perception mechanisms fail, and robots could be forced to adopt the approach of one "walking in the dark," touching surrounding objects toward the destination. Then convergence (which can be understood, for instance, as the robot reaching humans trapped under ruins or collapsed mine tunnels in finite time), is more important than protecting the hardware from collisions with the environment.

A challenging aspect of the problem at hand is that it consists of two different and equally difficult subproblems: the robot should plan and control its motion, and recover from unexpected (or avoid expected) collisions with objects. In the latter case, such objects may not be considered compliant when compared to, say, cardboard, but wood or plaster will definitely be more compliant than steel. Our approach is to bring together two different 
methodologies, each coping with one of these two particular subproblems, into a single, coherent, and provably convergent robot control scheme.

The control scheme we present in this paper is hybrid. It combines a component that aligns the robots velocity along the field produced by an artificial potential function, with a component that regulates contact force, using measurements provided by a force/torque sensor. Besides controlling velocity, instead of position, our hybrid scheme is different from what is widely known as "hybrid force/position control," introduced in ${ }^{35}$; the proposed hybrid scheme has two distinct modes of operation, and there is intermittent switching between the two, when specific conditions (guards) are satisfied. Not only do we have different closed loop dynamics different in each mode, but the state vectors are also different.

The contribution of this work is the use of force sensors for robot navigation in an unknown environment, offering guarantees of convergence to a desired configuration and recovery from collisions. We regard collisions not as undesirable events, but as opportunities to learn about the environment and discover a path that will bring the system closer to its goal. Our stability analysis not only ensures that the system will recover safely, but that it will also reach its destination, if a feasible path exists and the physical limitations of the mechanism are not exceeded.

\subsection{Related Work}

Hybrid systems ${ }^{30,3}$ are characterized by the interaction of continuous dynamics and discrete-valued dynamics. Typically, the continuous dynamics describes the system at a detailed concrete level, while some discrete logic 
(utilizing both discrete and continuous variables) regulates the system at a higher level. Ensuring stability in hybrid systems (especially during switching) is very important, since it is well known that switching between stable systems can result to instability ${ }^{25}$. There is considerable work in the area of stability for hybrid systems ${ }^{25}$, and one of the prevailing techniques is that of multiple Lyapunov functions ${ }^{4}$. This is the method of choice for us to establish the stability of our hybrid control scheme.

In the robotics literature, "hybrid force control" typically refers to the superposition of position and force control signals, in an approach initially proposed by ${ }^{35}$. Hybrid position/force controllers partition the task-space into position controlled directions and force controlled directions using natural constraints. The latter is a concept introduced in Reference ${ }^{31}$ : along the normal directions of these surfaces, the velocity is zero (position constraint), while the force is zero along tangential directions (force constraint). Specifying a desired velocity along an unconstrained direction and/or a desired force along a constrained direction gives rise to an artificial constraint. The basic idea of the hybrid force/position approach is to enforce only the artificial constraints. Reference ${ }^{8}$ raised questions about the orthogonality assumption between position and force controlled subspaces. Literature is rich with extensions of the original hybrid force/position scheme that modify the orthogonality condition and improve the overall system performance, such as the Dynamic Hybrid Force/Position Control ${ }^{43}$, the Operational Space Control $^{15}$, the Hybrid Impedance Control ${ }^{41}$, and the Hybrid scheme with force sensor ${ }^{10}$.

The latter approach ${ }^{10}$ utilizes force sensor measurements to partition the 
workspace into force and position controlled directions. The controller of The parallel force/position control ${ }^{6,38}$ combines the force and position controller into a single control law, so that unpredicted contact forces are accommodated. The parallel scheme has inspired in part our approach. Our original ambition was to extend this methodology so that motion planning can be done within the control loop. However, we had to abandon the idea of combining the motion (velocity) and force controller into a single construction because of stability concerns.

Besides force sensors, other sensors such as sensitive skins ${ }^{28}$, vision ${ }^{9}$ and strain gauges ${ }^{1}$ are also used for collision detection. Given an accurate robot dynamics model, it is possible to detect collision by comparing the actual torque (based on actuator currents) with the model-based calculated torque $^{39}$. The authors in ${ }^{27}$ use an approach for collision detection that relies on the robot dynamical model, without making use of sensors. However, since the exact magnitude and the direction of the contact force cannot be identified, the obstacle location cannot be determined.

The problem of navigating in an unknown environment is first addressed in $^{29}$, with application to mobile robots, without addressing the issue of integrating path-planning to lower level robot control. Behavior based control ${ }^{5,2}$ is an another approach for robot navigation in unstructured environments, where a specific behavior is reactively selected from a pre-defined set based on sensor information. ${ }^{44}$ have combined fuzzy logic controllers with such reactive behavioral schemes ${ }^{19,21,23}$, to provide a smooth transition between different behaviors and prevent unstable oscillations, and integrate multiple sensors ${ }^{20}$. Low-level reactive control is combined with high-level path-planning by ${ }^{22}$ us- 
ing a neural network.

An inherent limitation of reactive approaches is that it is extremely difficult to predict the overall system behavior resulting from the superposition of several elementary behaviors. Navigation functions ${ }^{36}$, however, produce artificial potential fields which do not have from local minima, a known limitation of the original potential field $\operatorname{method}^{16}$, and can therefore offer formal convergence guarantees. Performance comes at a price, and the assumption of a perfectly known environment prevents the direct application of this method to the problem at hand. To address this issue, article ${ }^{26}$, presents a kinematic controller based on a navigation function, which could steer a mobile robot in unknown environments using range sensors. The controller switches when an obstacle is detected. Our approach is along this line of thought, with the difference being that instead of using range sensors, the robot will have to physically touch the obstacle and recover from the collision.

\section{Problem Statement and Assumptions}

We seek a control scheme that can be applied to planar mobile robots and redundant manipulators equipped with the force sensors, alike. This control law should enable the robot to:

- Converge to the destination configuration, and

- Regulate contact forces while navigating amongst known and unknown obstacles. 
The robot is assumed to be described by dynamic equations of the form:

$$
M(x) \ddot{x}+C(x, \dot{x}) \dot{x}+G(x)=u-f,
$$

where $x \in \mathbb{R}^{2}$ is the vector of the mobile robot's or the manipulator's end effector position; $M(x) \in \mathbb{R}^{2 \times 2}$ is the inertia matrix; $C(x, \dot{x}) \in \mathbb{R}^{2 \times 2}$ is the matrix of Coriolis and centrifugal force terms; $G(x) \in \mathbb{R}^{2}$ is the vector of gravitational terms; $u \in \mathbb{R}^{2}$ is the input force vector, and $f \in \mathbb{R}^{2}$ is the force applied to the end effector or to the mobile robot's surface by the environment. The reason why we restrict the present analysis to the planar case will be explained in Section 3.3.

Given destination configuration $x_{D}$ in the task-space of a robot, the dynamics of which are given by (1), we need to find a feedback control law $u(x)$ such that:

- the robot approaches $x_{D}$ asymptotically from almost ${ }^{1}$ all initial configurations if $x_{D}$ is reachable, and

- the contact forces are bounded to a desired level.

We assume that the robots can obtain force measurements by means of force/torque sensors. A mobile robot is assumed to have a force sensor behind its front bumper. A manipulator is supposed to have a force sensor at its end effector. In the latter case, note that collisions can occur between intermediate arm links and obstacles, even if no force is measured at the end effector. This is an inherent limitation, resulting from our assumption that the robot uses a single force sensor. Future extensions of this work include

\footnotetext{
${ }^{1}$ The word "almost" allows the exclusion of a subset of the space with measure zero.
} 
modifications to the navigation scheme, so explored workspace safety is taken into account in motion planning.

The obstacles in the environment are assumed to occupy compact regions of the workspace, and their surface is smooth with no sharp edges. Mobile robots, on the other hand, are assumed as point objects. Sphere approximations of mobile robots shapes can be directly accounted for by "growing" the obstacles by the corresponding radius ${ }^{18}$. Manipulators are represented in the workspace as a series of control points along their links. In this approach, collisions between manipulator links and obstacles are in theory possible; a more thorough approach to real-time collision avoidance for multi-link mech-

anisms, has to take into account the whole volume of the robot ${ }^{42}$. It is possible to use a formulation such as the one described by ${ }^{42}$ with the proposed approach, but this is beyond the scope of this work. Our focus here is on control design and stability. Any improvement on the collision prediction component of our strategy will only bring benefits in terms of safety.

\section{Force/Potential Field Control}

Given a fairly accurate model of the system, (1) can be transformed into those of a linear second order system:

$$
\ddot{x}=u_{s},
$$

by means of a feedback linearizing input:

$$
u=M(x) u_{s}+C(x, \dot{x}) \dot{x}+G(x)+f,
$$




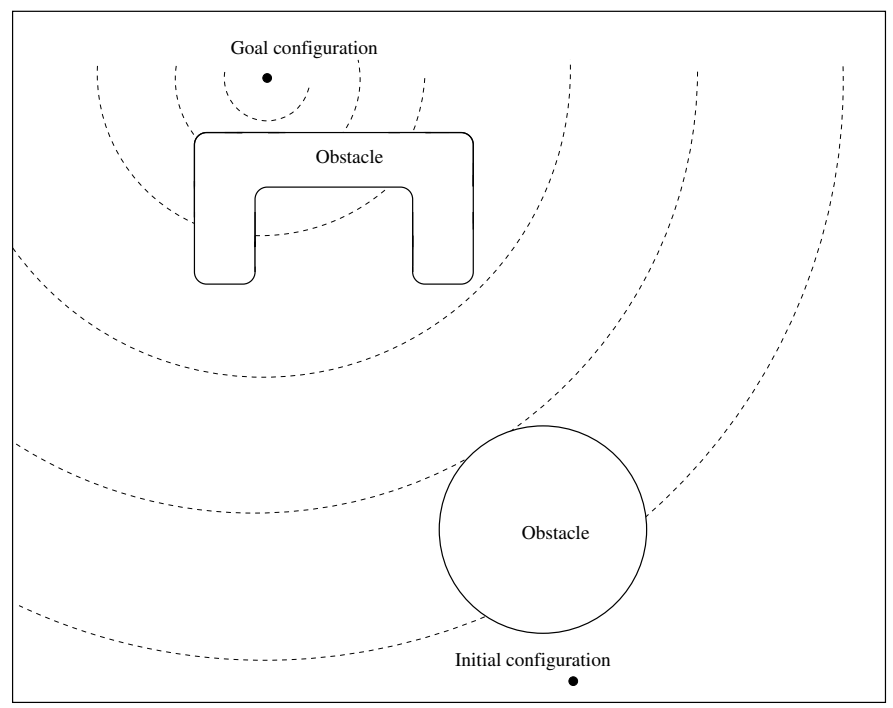

Figure 1: An example of a navigation problem in 2-D workspace: the robot has to move from initial configuration to goal, without prior knowledge of the two obstacles' presence. Dotted lines denote points of equal distance to destination.

where $u_{s}$ denotes the new input to the linearized system. While navigating, the robot executes either free motion, having no contact with the environment, or constraint motion, when in contact with an obstacle. Each case is considered as a separate (discrete) mode in the following simple hybrid automaton of Figure 2. A general formal definition of hybrid automaton is as follows:

Definition $1\left({ }^{30}\right)$. A hybrid automaton is a collection $\mathcal{H}=\{\mathcal{Q}, \mathcal{X}, \mathcal{F}$, Init, $\mathcal{D}, \mathcal{E}, \mathcal{G}, \mathcal{R}\}$, where

- $\mathcal{Q}=\left\{q_{1}, q_{2}, \ldots\right\}$ is a set of discrete states;

- $\mathcal{X} \subseteq \mathbb{R}^{n}$ is a set of continuous states; 


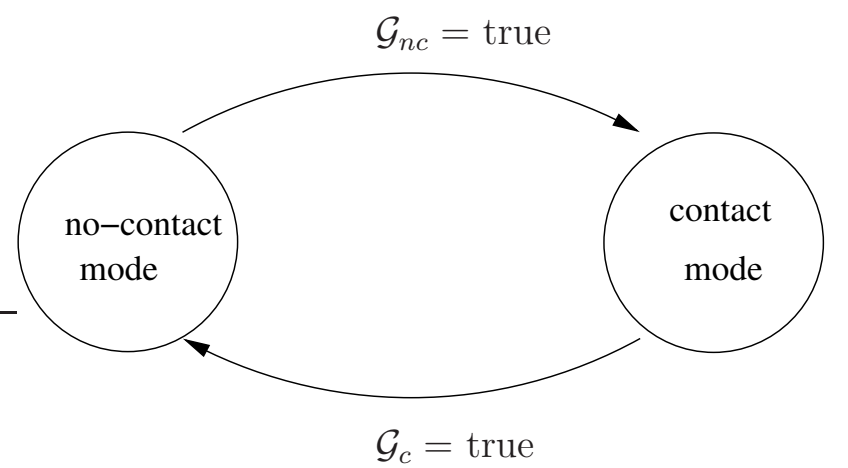

Figure 2: The hybrid system describing the closed loop dynamics of the system. The predicates $G_{n c}$ and $G_{c}$ correspond to the guards on the nocontact and contact modes, respectively, that force transitions. Part of the control design problem is to define $G_{n c}$ and $G_{c}$.

- $\mathcal{F}(\cdot, \cdot): \mathcal{Q} \times \mathcal{X} \rightarrow \mathbb{R}^{n}$ is a vector field;

- Init $\subseteq \mathcal{Q} \times \mathcal{X}$ is a set of initial states;

- $\mathcal{D}: \mathcal{Q} \rightarrow P(\mathcal{X})$, is a domain;

- $\mathcal{E} \subseteq \mathcal{Q} \times \mathcal{Q}$ is a set of edges;

- $\mathcal{G}: \mathcal{E} \rightarrow P(\mathcal{X})$ is a guard;

- $\mathcal{R}: \mathcal{E} \times \mathcal{X} \rightarrow P(\mathcal{X})$ is a reset map.

The set $P(\cdot)$ denotes the power set (set of all subsets). We refer to $(q, x) \in$ $\mathcal{Q} \times \mathcal{X}$ as the state of $\mathcal{H}$. 


\subsection{No-Contact Mode}

During free motion, the robot follows the negated gradient of a navigation function, constructed on the known workspace. To account for the nonpoint, multi-link structure of the manipulator, we define control points along its links. Proximity of the robot to obstacles is assessed by considering all distances between control points and workspace obstacles. Our navigation function has the following form ${ }^{17}$

$$
\phi=\frac{\gamma(x)}{\exp \left(\beta(x)^{1 / \kappa}\right)}
$$

The terms involved in the above expression are defined as follows:

- Function $\beta$ is the product of several functions,

$$
\beta=\prod_{\substack{c=1, \ldots, p \\ o=0, \ldots, q}} \beta_{c o},
$$

each encoding the proximity between a control point on the system $c$, and a known obstacle $o$. These functions are given $\operatorname{as}^{17} \beta_{c o}=(1-$ $\left.\lambda \frac{\left(\left\|x_{c}-x_{o}\right\|^{2}-d^{2}\right)^{4}}{\left(\left\|x_{c}-x_{o}\right\|^{2}-d^{2}\right)^{4}+1}\right)^{\frac{\operatorname{sign}\left(d-\left\|x_{c}-x_{o}\right\|\right)+1}{2}}$, with $d$ being the distance to which the presence of the obstacle is being "felt" by the robot, and $\lambda=\frac{1+d^{8}}{d^{8}}$.

The workspace boundary is taken into account as an additional obstacle (obstacle 0) and is expressed as $\beta_{c 0}=\left(1-\lambda \frac{\left.\left(\left\|x_{c}\right\|-R\right)^{2}-d^{2}\right)^{4}}{\left.\left(\left\|x_{c}\right\|-R\right)^{2}-d^{2}\right)^{4}+1}\right)^{\operatorname{sign}\left(d-\left(R-\left\|x_{o}\right\|\right)\right)+1} \frac{1}{2}$. The part of the workspace occupied by obstacles is defined as $\mathcal{B} \triangleq\left\{x_{c} \in\right.$ $\left.\mathbb{R}^{2} \mid \beta\left(x_{c}\right) \leq 0\right\}$.

- Function $\gamma$ is a nonnegative function that encodes the proximity to the destination configuration, $\gamma(x)=\left\|x-x_{D}\right\|^{2}$. 
- Parameter $\kappa$ is a tuning constant, which is adjusted based on the geometry of the (known) workspace according to ${ }^{17}$, to make (4) a navigation function.

Obstacle functions are equal to one in the region where $\left\|x_{c}-x_{o}\right\|>d$, thus making the effect of obstacles local. Thus, obstacle points can potentially be included "on the fly" into the navigation function, and the tuning parameter $\kappa$ can be adjusted accordingly, without having to change the structure of $\varphi$.

The potential field based controller used for steering the robot during the no-contact phase of its motion is given as:

$$
u_{s}=-\mathrm{K}_{D} \dot{x}-\nabla \phi
$$

where $-\nabla \phi$ is the negative gradient of (4), and $K_{D}>0$ is the gain of the viscous damping term.

\subsection{Contact Mode}

When a collision between the robot and an obstacle occurs, contact forces are exerted by the obstacle to the robot, which are assumed normal to the obstacle surface. A force sensor measures the contact forces along different directions: $-\hat{f}=\left[\hat{\mathrm{f}}_{1}, \hat{\mathrm{f}}_{2}\right]^{T}$. The force that the robot exerts on the the object can be expressed compactly as $\hat{f}=\mathrm{f} \eta$, where $\mathrm{f} \triangleq\|\hat{f}\|$ is the measured force's magnitude, and $\eta \triangleq-\frac{1}{\mathrm{f}} \hat{f}$ for $\mathrm{f} \neq 0$ denotes the direction, normal to the obstacle's surface, along which the robot exerts a force. A tangential direction is chosen to form an orthonormal basis in $\mathbb{R}^{2}: \tau \in \operatorname{span}(\eta)^{\perp}$, and $[\eta, \tau] \in S O(2)$. This coordinate frame will be called the constraint frame. 


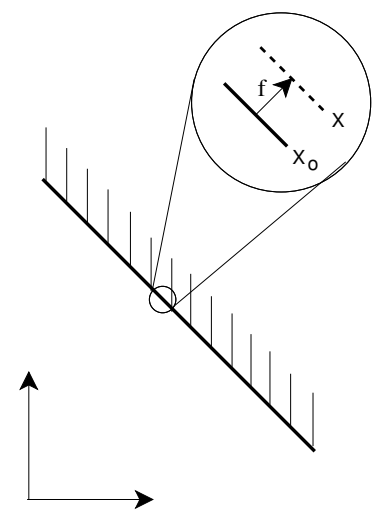

Figure 3: Force exerted to the obstacle surface resulting to local deformation according to the contact model (7).

We adopt a linear spring contact model, which describes an obstacle as a compliant, frictionless surface:

$$
\mathrm{f}=\mathrm{K}\left(\mathrm{x}-\mathrm{x}_{0}\right)
$$

where $\mathrm{x} \in \mathbb{R}$ is the position of the contact point along $\eta, \mathrm{x}_{0} \in \mathbb{R}$ is the position, along $\eta$, the contact point would have if the obstacle surface were undeformed, and $K \in \mathbb{R}$ is the stiffness constant of the surface. High obstacle stiffness allows us to neglect local deformations at the contact point. Equation (7) applies along the normal to the surface direction.

Figure 3 shows the contact force due to surface deformation. The compliant contact model $(7)$ is used to express the desired contact force, $f_{d}$ :

$$
\mathrm{f}_{d}=\mathrm{K}\left(\mathrm{x}_{f_{d}}-\mathrm{x}_{0}\right)
$$

where $\mathrm{x}_{f_{d}} \in \mathbb{R}$ is the position of the end effector along $\eta$, producing the desired contact force $\mathrm{f}_{d}$ according to (7). Combining (7) with (8):

$$
\Delta \mathrm{f}=\mathrm{f}_{d}-\mathrm{f}=\mathrm{K}\left(\mathrm{x}_{f_{d}}-\mathrm{x}\right) .
$$


The control law for the contact mode is defined as follows ${ }^{40}$ :

$$
u_{s}=-\underbrace{\mathrm{K}_{D} \dot{x}}_{\text {damping }}+\underbrace{\mathrm{K}_{D} \mathrm{v}_{d} \tau}_{\text {velocity feedforward }}+\underbrace{\mathrm{K}_{F} \Delta \mathrm{f} \eta+\mathrm{K}_{I} \int_{t_{1}}^{t_{2}} \Delta \mathrm{f} \mathrm{d} \sigma \eta}_{\text {force control }},
$$

where $\mathrm{v}_{d}$ is a constant reference speed in the tangential direction. The limits

of the integral $t_{1}$, and $t_{2}$ are the time instants when the contact was initiated and terminated, respectively. Parameters $\mathrm{K}_{D}, \mathrm{~K}_{F}$, and $\mathrm{K}_{I}$ are positive scalar feedback gains.

Along the normal direction $\eta$, the control law (10) attempts to stabilize the contact force to the reference $\mathrm{f}_{d}$. A nonzero $\mathrm{f}$ maintains contact between the robot and the obstacle is maintained. Along the tangential direction $\tau$ the feedforward and damping terms, form a PD velocity controller, which stabilizes the end effector velocity $\dot{x}$ to the reference $\mathrm{v}_{d}$.

\subsection{Switching Conditions}

The detection of a nonzero contact force marks the transition from free motion to contact with an obstacle. This event forces a control law switch, from (6) to (10), to alleviate the effects of the collision. The transition to free motion, however, cannot be arbitrary because the stability may be affected.

Let us denote $x_{h}$ the point on the obstacle boundary where contact is initiated. Let $x_{e}$ be the point at which the transition from contact-mode control to no-contact-mode control is to take place. The (forced) "guard" that triggers the transitions from contact to no-contact will include:

$$
\left(\phi\left(x_{e}\right) \leq \phi\left(x_{h}\right)\right) \wedge\left(-\nabla \phi\left(x_{e}\right)^{T} \eta<0\right) .
$$




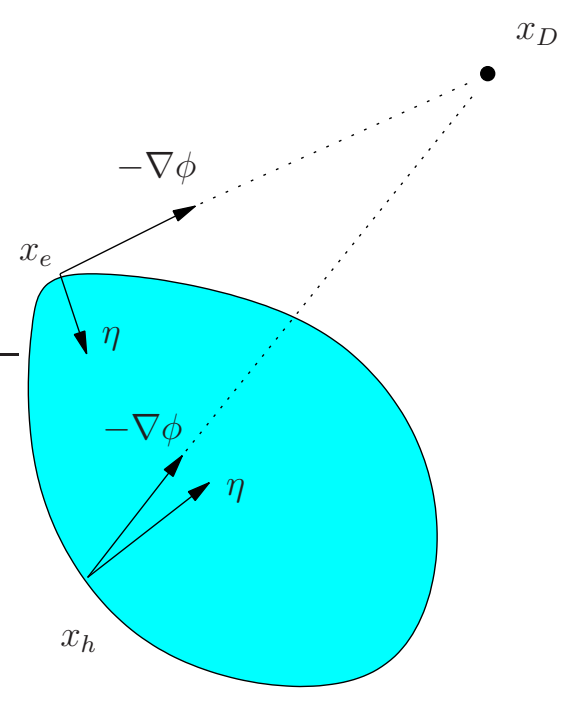

Figure 4: The contact entry and exit points, $x_{h}$ and $x_{e}$, on obstacle's surface, and relative alignment with the potential field gradient.

The first condition in (11) ensures that the system's configuration at $x_{e}$ is "closer" to the destination than the configuration $x_{h}$ where contact was initiated (as measured by the potential function $\phi$ ). Thus, each time the system is reset to no-contact mode, the value of $\phi$ is decreased compared to all values $\phi$ has obtained previously during free motion. The second condition requires that the obstacle surface normal is (partially) aligned with the direction for free motion, $-\nabla \phi$. This will indicate that the obstacle is not obstructing the path to destination (at least locally), and that the system can move in the free space for at least some minimum (dwell) time before switching back to contact mode. The condition, illustrated in Figure 4, eliminates the possibility of Zeno executions in the hybrid system.

For the class of obstacles considered, the transition from contact to nocontact mode happens in finite time: 
Proposition 1. In a two-dimensional workspace, a system (2) under control law (10) will satisfy $\mathcal{G}_{c}$ in finite time.

Proof. The control law (10) forces the system to slide continuously, along a curve $c(t)$ on the obstacle's boundary. Since each obstacle defines a compact set, $\mathcal{B}$, its boundary $\partial \mathcal{B}$ and any continuous curve on it, $c(t) \in \partial \mathcal{B}$, is also compact. The continuous function $\phi$ will therefore assume a minimum value $\phi\left(x_{b}\right)$ at $c(t)=x_{b}$. Sliding along the obstacle boundary with a nonzero speed, the robot will eventually reach $x_{b}$, satisfying the first condition in (11). Note that $x_{b}$ minimizes $\phi$ globally on $\mathcal{B}$, because the destination is not covered by the obstacle - otherwise it would be unreachable. If $-\nabla \phi$ pointed inward at $x_{b}$, there would be points in $\mathcal{B}$ with smaller values of $\phi$ than $\phi\left(x_{b}\right)$; a contradiction. Therefore, $-\nabla \phi$ points outward at $x_{b}$. Since $\eta$ points inward, ( $\eta$ is the direction along which the robot exerts a force on the obstacle), we will have $-\nabla \phi\left(x_{b}\right)^{T} \eta\left(x_{b}\right)<0$. Thus at $x_{b}$, the second inequality in (11) will also be satisfied.

\subsection{Redundancy Resolution in Contact Mode}

In the control schemes of the previous sections, only the task space coordinates of the systems are being controlled. Depending on the degree of redundancy, the system may possess additional degrees of freedom. In this section, redundancy is exploited so that links of the redundant manipulator avoid collisions with known (or newly discovered) obstacles. We need to meet two objectives: (i) maintain contact between the end effector and the obstacles; (ii) avoid contact between the arm links and already detected obstacles. Simultaneously satisfying both objectives may be infeasible, and 
therefore they have to be prioritized. In this approach, maintaining contact between end-effector and obstacle is assigned higher priority, since the focus is on convergence of the robot to the destination. The task-space acceleration $\ddot{x}$ can be written in terms of joint space coordinates $\theta$ as $\ddot{x}=J \ddot{\theta}+\dot{j} \dot{\theta}$, where $\ddot{\theta} \in \mathbb{R}^{n}$ and $\dot{\theta} \in \mathbb{R}^{n}$ are joint space acceleration and velocity, respectively, and $J \in \mathbb{R}^{2 \times n}$ is the manipulator's Jacobian, which is a function of $\theta$.

It is well known ${ }^{33,11}$ that redundancy resolution schemes resolved at the acceleration level, can cause divergence and instability because of built-up of joint space velocities. We circumvent this problem by including an integrator in our task space control law (10), to generate reference task space velocities $\dot{x}_{d}$, instead of accelerations:

$$
\dot{x}_{d}=\int_{t_{1}}^{t_{2}}\left[-\mathrm{K}_{D} \dot{x}+\mathrm{K}_{D} \mathrm{v}_{d} \tau+\left(\mathrm{K}_{F} \Delta \mathrm{f}+\mathrm{K}_{I} \int_{t_{1}}^{t_{2}} \Delta \mathrm{f} \mathrm{d} \sigma\right) \eta\right] \mathrm{d} t .
$$

Now the task space reference velocity $\dot{x}_{d}$ is given in joint space coordinates using the general solution involving the pseudoinverse ${ }^{32}$ of $J, J^{\dagger}$ :

$$
\dot{\theta}_{d}=J^{\dagger} \dot{x}_{d}+\left(I-J^{\dagger} J\right) h
$$

where $\left(I-J^{\dagger} J\right)$ is the projection matrix to the null space of $J$, and $h$ is a vector utilized to encode the secondary objective:

$$
h=\mathrm{K}_{o} \frac{\partial \beta(x)}{\partial \theta}
$$

where $\beta(x)$ is the obstacle function defined in (5) and $\mathrm{K}_{o}$ is the positive gain. The reference joint space acceleration is now defined as:

$$
\ddot{\theta}_{d}=J^{\dagger}\left(\ddot{x}_{d}-\dot{J} \dot{\theta}_{d}\right)
$$


The manipulator torques needed to realize this reference acceleration is:

$$
\mathcal{T}=M(\theta)\left(\ddot{\theta}_{d}+\mathrm{K}_{v j}\left(\dot{\theta}_{d}-\dot{\theta}\right)\right)+C(\theta, \dot{\theta}) \dot{\theta}+G(\theta)+J^{T} f
$$

where $\mathrm{K}_{v j}$ is the positive gain, yielding the closed loop joint space dynamics:

$$
\left(\ddot{\theta}_{d}-\ddot{\theta}\right)+\mathrm{K}_{v j}\left(\dot{\theta}_{d}-\dot{\theta}\right)=0 .
$$

\section{Continuous Closed Loop Dynamics}

\subsection{Contact Mode}

Using (9), the closed loop system during contact (10) is expressed in standard state-space form:

$$
\underbrace{\left[\begin{array}{c}
\dot{\mathrm{x}}_{1} \\
\dot{x}_{2} \\
\dot{\mathrm{x}}_{3}
\end{array}\right]}_{\dot{y}}=\underbrace{\left[\begin{array}{ccc}
0 & -\eta^{T} & 0 \\
\mathrm{~K}_{F} \mathrm{~K} \eta & -\mathrm{K}_{D} & \mathrm{~K}_{I} \mathrm{~K} \eta \\
1 & \mathbf{0}^{T} & 0
\end{array}\right]}_{A} \underbrace{\left[\begin{array}{l}
\mathrm{x}_{1} \\
x_{2} \\
\mathrm{x}_{3}
\end{array}\right]}_{y},
$$

where $\mathbf{0}$ denotes the $(3 \times 1)$ vector of zeros. Symbols $\mathrm{x}_{1}, \mathrm{x}_{3}$, and $x_{2}$ express the coordinates of a $(4 \times 1)$ state vector $y$. They are given as $\mathrm{x}_{1} \triangleq \mathrm{x}_{f_{d}}-\mathrm{x}$, the force error translated into displacement through the contact model; $x_{2} \triangleq \dot{x}-\mathrm{v}_{d} \tau$, the velocity error; and $\mathrm{x}_{3} \triangleq \int_{t_{1}}^{t_{2}} \mathrm{x}_{1} \mathrm{~d} \sigma$, the force error integral.

Equation (17) describes the closed loop system in the case of mobile robots, but for robotic manipulators redundancy resolution imposes different dynamics on the task and joint spaces. The task-space control law defined in (10) ensures that contact between the robot and the obstacle is maintained; the joint-space control law (16) implements (10) by performing collision avoidance in the null space of the manipulator's Jacobian. Combining 
(16), (10) yields the closed loop system dynamics:

$$
\begin{aligned}
{\left[\begin{array}{c}
\dot{\mathrm{x}}_{1} \\
\dot{x}_{2} \\
\dot{\mathrm{x}}_{3}
\end{array}\right] } & =\left[\begin{array}{ccc}
0 & -\eta^{T} & 0 \\
\mathrm{~K}_{F} \mathrm{~K} \eta & -\mathrm{K}_{D} & \mathrm{~K}_{I} \mathrm{~K} \eta \\
1 & \mathbf{0}^{T} & 0
\end{array}\right]\left[\begin{array}{l}
\mathrm{x}_{1} \\
x_{2} \\
\mathrm{x}_{3}
\end{array}\right]+\left[\begin{array}{c}
0 \\
\mathrm{~K}_{v j}\left(\dot{\theta}_{d}-\dot{\theta}\right) \\
0
\end{array}\right] \\
\dot{\theta}_{d} & =J^{\dagger}\left(\dot{x}_{d}+\mathrm{v}_{d} \tau\right)+\left(I-J^{\dagger} J\right) h
\end{aligned}
$$

where $\dot{x}_{d}$ and $h$ are given by (12) and (14), respectively. Equation (18) describes the dynamics of a linear system that is perturbed by the term $\mathrm{K}_{v j}\left(\dot{\theta}_{d}-\dot{\theta}\right)$. As a prelude of what follows, we will regard this term as a perturbation term, that will be made to converge to zero sufficiently fast.

\subsection{No-Contact Mode}

In no-contact mode, no force-related state variables are defined. Since there is no contact, $\mathrm{f}_{d}=0$ and therefore $\dot{x} \equiv x_{2}$. Equations (3), (6) yield the following closed loop system:

$$
\dot{x}_{2}=-\mathrm{K}_{D} x_{2}-\nabla \phi(x)
$$

\section{$5 \quad$ Stability Analysis}

Lyapunov stability theory for switched systems imposes conditions not only on each admissible dynamics, but also on the switching signal ${ }^{25,4,13,12}$. This Section refines the switching conditions of Section 3.3 to establish the stability of the hybrid system defined in Section 3. We follow a multiple Lyapunov function approach. 


\subsection{Contact Mode}

The following proposition states that the (unperturbed) closed loop dynamics of the contact mode (that is, equation (17)) is exponentially stable:

Proposition 2. There exists a choice of gains $\mathrm{K}_{D}, \mathrm{~K}_{F}, \mathrm{~K}_{I}$ that makes the origin of the system (17) exponentially stable.

Proof. Consider the Lyapunov function candidate for (17) :

$$
V_{c}=\frac{1}{2} y^{T} P y
$$

where $P$ is the symmetric matrix defined as:

$$
P \triangleq\left[\begin{array}{ccc}
\mathrm{K}_{D}+\mathrm{K}_{F} \mathrm{~K}-1 & -\eta^{T} & \mathrm{~K}_{I} \mathrm{~K}+\mathrm{K}_{D} \\
-\eta & I & -\eta \\
\mathrm{K}_{I} \mathrm{~K}+\mathrm{K}_{D} & -\eta^{T} & \mathrm{~K}_{I} \mathrm{~K}+\mathrm{K}_{F} \mathrm{~K}
\end{array}\right]
$$

and $\mathrm{K}_{D}, \mathrm{~K}_{F}, \mathrm{~K}$ are positive scalars. Expanding (20):

$V_{c}=\frac{1}{2}\left[\begin{array}{l}\mathrm{x}_{1} \\ x_{2}\end{array}\right]^{T}\left[\begin{array}{cc}\mathrm{K}_{D}-1 & -\eta^{T} \\ -\eta & \frac{1}{2} I\end{array}\right]\left[\begin{array}{l}\mathrm{x}_{1} \\ x_{2}\end{array}\right]+\frac{\mathrm{K}}{2}\left[\begin{array}{l}\mathrm{x}_{1} \\ \mathrm{x}_{3}\end{array}\right]^{T}\left[\begin{array}{ll}\mathrm{K}_{F} & \mathrm{~K}_{I} \\ \mathrm{~K}_{I} & \mathrm{~K}_{I}\end{array}\right]\left[\begin{array}{l}\mathrm{x}_{1} \\ \mathrm{x}_{3}\end{array}\right]+\frac{1}{2}\left[\begin{array}{l}x_{2} \\ \mathrm{x}_{3}\end{array}\right]^{T}\left[\begin{array}{cc}\frac{1}{2} I & -\eta \\ -\eta^{T} & \mathrm{~K}_{F} \mathrm{~K}\end{array}\right]\left[\begin{array}{l}x_{2} \\ \mathrm{x}_{3}\end{array}\right]$.

If we assume that

$$
\mathrm{K}_{D}>2 \lambda_{\max }+1=3, \quad \mathrm{~K}_{F}>\mathrm{K}_{I}+\frac{\mathrm{K}_{D}}{\mathrm{~K}}, \quad \mathrm{~K}_{F}>\frac{2}{\mathrm{~K}},
$$

where $\lambda_{\max }=1$ is the maximum eigenvalue of $\eta \eta^{T}$, then each matrix in (22) will be positive definite. Then there will be $c_{1}$ and $c_{2}$ for which $c_{1}\|y\|^{2} \leq$ $V_{c} \leq c_{2}\|y\|^{2}$. The derivative of $V_{c}$ along (17) is:

$$
\dot{V}_{c}=-\left(\mathrm{K}_{F} \mathrm{~K}-\mathrm{K}_{I} \mathrm{~K}-\mathrm{K}_{D}\right) \mathrm{x}_{1}^{2}-x_{2}^{T}\left(\mathrm{~K}_{D} I-\eta \eta^{T}\right) x_{2}-\mathrm{K}_{I} \mathrm{Kx}_{3}^{2}<0,
$$


which is negative definite due to (23). Then (24) yields:

$$
\begin{aligned}
\dot{V}_{c} \leq & -\left(\mathrm{K}_{F} \mathrm{~K}-\mathrm{K}_{I} \mathrm{~K}-\mathrm{K}_{D}\right) \mathrm{x}_{1}{ }^{2}-\left(\mathrm{K}_{D}-\lambda_{\max }\right)\left\|x_{2}\right\|^{2}-\mathrm{K}_{I} \mathrm{Kx}_{3}^{2} \\
& -\min \left\{\mathrm{K}_{F} \mathrm{~K}-\mathrm{K}_{I} \mathrm{~K}-\mathrm{K}_{D}, \mathrm{~K}_{D}-\lambda_{\max }, \mathrm{K}_{I} \mathrm{~K}\right\}\|y\|^{2},
\end{aligned}
$$

which establishes the exponential stability of (17).

\section{$5.2 \quad$ No-Contact Mode}

By means of an appropriate Lyapunov function, we show that in the nocontact mode, the destination configuration is an almost globally asymptotically stable equilibrium of the closed loop dynamics . The characterization "almost" is included to exclude a subset of the state space with measure zero, that contains unstable equilibria ${ }^{36}$ :

Proposition 3. If $\mathrm{K}_{D}>0$, then the system (19) is (almost globally) asymptotically stable at the destination.

Proof. Consider $V_{n c}=\frac{1}{2}\|\dot{x}\|+\phi(x)$ as Lyapunov function candidate for (19). The derivative of $V_{n c}$ along the trajectories of (19) is:

$$
\dot{V}_{n c}=-\mathrm{K}_{D}\|\dot{\mathrm{x}}\|^{2}-\dot{x}^{T} \nabla \phi+\nabla \phi^{T} \dot{x}=-\mathrm{K}_{D}\|\dot{\mathrm{x}}\|^{2}
$$

which is negative semi-definite because $\mathrm{K}_{D}>0$. Note that $V_{n c}$ is positive definite, since $\phi(x)$ is positive everywhere except for the destination $x_{D}$. Its levels sets, therefore, define compact subsets of the state space, which are also invariant due to (25). By the invariance principle, the system converges to the largest invariant set in the region where $\dot{V}_{n c}(x)=0 \Rightarrow \dot{x}=0$ for all $t>0$. The dynamics in this set are obtained from (19) substituting for 
$\dot{x}=\ddot{x}=0: \nabla \phi(x)=0$, which is true at $x_{D}$ and a set of isolated (unstable) critical points of the navigation function $\phi$. The system converges to these unstable equilibria only by flowing along a set of measure zero.

\subsection{Switching Conditions for Stability}

To establish the stability of the closed loop hybrid system depicted in Figure 2, we will apply a classic result in switched systems concerning multiple Lyapunov functions ${ }^{4}$. A switching system is defined as a collection of subsystems $^{24}: \dot{x}=f_{\sigma}(x)$, where $\sigma[0, \infty) \rightarrow \mathcal{P}$ is a piecewise constant function taking values in a finite subset $\mathcal{P} \subset \mathbb{N}$. The swiching function $\sigma(t)$ indicates which subsystem, say $p$, is active at time $t$ :

$$
\dot{x}(t)=f_{p}(x(t)) .
$$

The stability of the switched system (26) can be established as follows:

Theorem $4\left({ }^{4}\right)$. If for each $p$ in $\mathcal{P}$ the system (26) is asymptotically stable, i.e., for each $p, x(t) \rightarrow 0$ as $t \rightarrow \infty$ when $\dot{x}(t)=f_{p}(x(t)), \quad \forall t \geq t_{0}$, and there is a family of Lyapunov functions, $V_{p}$, for all $p$, such that for any two switching times $t_{j}$ and $t_{k}$ with $j<k$ we have:

$$
V_{p}\left(x\left(t_{j}\right)\right)-V_{p}\left(x\left(t_{k}\right)\right)>0
$$

Then system $\dot{x}=f_{\sigma}(x)$ is asymptotically stable.

Let $T=\left\{t_{0}, t_{1}, t_{2}, \ldots\right\}$ be a strictly increasing sequence of switching times and let $\mathcal{N C}(T)(\mathcal{C}(T))$ denote the sequence of switching instants at which the system enters into no-contact mode (contact mode). If the system is initially in free space, $\mathcal{N C}(T)=\left\{t_{0}, t_{2}, t_{4}, \ldots\right\}$ and $\mathcal{C}(T)=\left\{t_{1}, t_{3}, \ldots\right\}$. 


\subsubsection{Switching from Contact to No-contact Mode}

According to Theorem 4, one of the conditions for the stability of a hybrid system is that the Lyapunov functions do not increase at switching instants. The following proposition establishes that the switching from contact to nocontact mode, does not allow the Lyapunov function of the no-contact control mode to increase, compared to its values at the previous switching instant.

Proposition 5. For any two switching times $t_{k} \in \mathcal{N C}(T)$ and $t_{j} \in \mathcal{C}(T)$ such that $k-j=1$, if (i) $\phi\left(x\left(t_{k}\right)\right)<\phi\left(x\left(t_{j}\right)\right)$, and (ii) $\left\|x_{2}\left(t_{k}\right)\right\|<\left\|x_{2}\left(t_{j}\right)\right\|$, then $V_{n c}\left(x\left(t_{j}\right)\right)-V_{n c}\left(x\left(t_{k}\right)\right)>0$.

Proof. Given that $\dot{x} \equiv x_{2}$ at switching instant $t_{k}$, for the Lyapunov function of the no-contact mode we have $V_{n c}\left(x\left(t_{k}\right)\right)=\frac{1}{2}\left\|x_{2}\left(t_{k}\right)\right\|^{2}+\phi\left(x\left(t_{k}\right)\right)<$ $\frac{1}{2}\left\|x_{2}\left(t_{j}\right)\right\|^{2}+\phi\left(x\left(t_{j}\right)\right)=V_{n c}\left(x\left(t_{j}\right)\right)$.

\subsubsection{Switching from No-contact to Contact Mode}

When an obstacle is detected by means of the force sensor, the control law switches from no-contact to contact mode. At switching instant $t_{j}$, the contact mode Lyapunov function in (22) becomes

$$
V_{c}\left(x\left(t_{j}\right)\right)=\left(\mathrm{K}_{D}+\mathrm{K}_{F} \mathrm{~K}-1\right) \mathrm{x}_{1}^{2}\left(t_{j}\right)-2 \mathrm{x}_{1}\left(t_{j}\right) \eta^{T} x_{2}\left(t_{j}\right)+\left\|x_{2}\left(t_{j}\right)\right\|^{2},
$$

since $\mathrm{x}_{3}\left(t_{j}\right)=0$ at $t_{j} \in \mathcal{C}(T)$. Obstacle surface deformation $\mathrm{x}_{1}$ depends on the impact velocity $x_{2}$. Conservation of energy during impact yields

$$
x_{2}^{T}\left(t_{j}\right) M x_{2}\left(t_{j}\right)=\mathrm{Kx}_{1}^{2}\left(t_{j}\right) \Rightarrow \mathrm{x}_{1}\left(t_{j}\right)=\sqrt{\frac{x_{2}\left(t_{j}\right)^{T} M x_{2}\left(t_{j}\right)}{\mathrm{K}}},
$$

and substituting (29) into (28) we obtain $V_{c}\left(x\left(t_{j}\right)\right)=\left(\mathrm{K}_{D}+\mathrm{K}_{F} \mathrm{~K}-1\right) \frac{x_{2}\left(t_{j}\right)^{T} M x_{2}\left(t_{j}\right)}{\mathrm{K}}-$ $2 \sqrt{\frac{x_{2}\left(t_{j}\right)^{T} M x_{2}\left(t_{j}\right)}{\mathrm{K}}} \eta^{T} x_{2}\left(t_{j}\right)+\left\|x_{2}\left(t_{j}\right)\right\|^{2}$. 
Proposition 6. For any two switching times $t_{j}, t_{k} \in \mathcal{C}(T)$ with $k>j$, if

$$
\frac{\left\|x_{2}\left(t_{k}\right)\right\|}{\left\|x_{2}\left(t_{j}\right)\right\|}<\frac{3 \underline{\lambda}(M)}{\sqrt{\mathrm{K}\left(\mathrm{K}_{D}+\mathrm{K}_{F} \mathrm{~K}-1\right) \bar{\lambda}(M)}+\mathrm{K}}
$$

where $\underline{\lambda}(M)$ is a lower bound on the smallest eigenvalue of $M$ and $\bar{\lambda}(M)$ is an upper bound on the largest eigenvalue of $M$, then $V_{c}\left(x\left(t_{j}\right)\right)-V_{c}\left(x\left(t_{k}\right)\right)>0$.

Proof. Conditions (23) imply that $\mathrm{K}_{D}+\mathrm{K}_{F} \mathrm{~K}-1>\mathrm{K}_{D}+1>4$. In view of this fact, $V_{c}$ at the contact instant can be lower bounded as follows (dropping the dependence on $t_{j}$ for brevity):

$$
\begin{aligned}
V_{c} & >\frac{4 x_{2}^{T} M x_{2}}{\mathrm{~K}}-2 \sqrt{\frac{x_{2}^{T} M x_{2}}{\mathrm{~K}}}\left\|x_{2}\right\|+\left\|x_{2}\right\|^{2} \\
& >\left(\sqrt{\frac{x_{2}^{T} M x_{2}}{\mathrm{~K}}}-\left\|x_{2}\right\|\right)^{2}+\frac{3 x_{2}^{T} M x_{2}}{\mathrm{~K}}>\frac{3 \bar{\lambda}_{\min }(M)}{\mathrm{K}}\left\|x_{2}\right\|^{2},
\end{aligned}
$$

where $\underline{\lambda}(M)$ is a lower bound on the minimum eigenvalue of the positive definite inertia matrix $M$. Similarly, defining $\bar{\lambda}(M)$ to be an upper bound on the maximum eigenvalue of $M$, we can bound $V_{c}\left(x\left(t_{j}\right)\right)$ from above:

$$
\begin{aligned}
V_{c} & <\frac{\left(\mathrm{K}_{D}+\mathrm{K}_{F} \mathrm{~K}-1\right) x_{2}^{T} M x_{2}}{\mathrm{~K}}+2 \sqrt{\frac{\left(\mathrm{K}_{D}+\mathrm{K}_{F} \mathrm{~K}-1\right) x_{2}^{T} M x_{2}}{\mathrm{~K}}}\left\|x_{2}\right\|+\left\|x_{2}\right\|^{2} \\
& <\left(\sqrt{\frac{\left(\mathrm{K}_{D}+\mathrm{K}_{F} \mathrm{~K}-1\right) \bar{\lambda}(M)}{\mathrm{K}}}+1\right)\left\|x_{2}\right\|^{2}
\end{aligned}
$$

Note now that if $(30)$ is true we can write: $V_{c}\left(t_{k}\right) \stackrel{(32)}{<}\left(\sqrt{\frac{\left(\mathrm{K}_{D}+\mathrm{K}_{F} \mathrm{~K}-1\right) \bar{\lambda}_{\max }(M)}{\mathrm{K}}}+1\right)$ $\left\|x_{2}\left(t_{k}\right)\right\|^{2} \stackrel{(30)}{<} \frac{3 \underline{\lambda}(M)}{\mathrm{K}}\left\|x_{2}\left(t_{j}\right)\right\|^{2} \stackrel{(31)}{<} V_{c}\left(t_{j}\right)$. 
We define the guards for the hybrid system of Figure 2 as follows:

$$
\begin{aligned}
G_{n c}: & \mathrm{f}_{d} \neq 0 \\
G_{c}: & \left\{\begin{array}{l}
\phi\left(x\left(t_{k}\right)\right)<\phi\left(x\left(t_{j}\right)\right), \\
-\eta\left(x\left(t_{k}\right)\right)^{T} \nabla \phi\left(x\left(t_{k}\right)\right)<0, \text { and } \\
\frac{\left\|x_{2}\left(t_{k}\right)\right\|}{\left\|x_{2}\left(t_{j}\right)\right\|}<\frac{3 \underline{\lambda}(M)}{\sqrt{\mathrm{K}\left(\mathrm{K}_{D}+\mathrm{K}_{F} \mathrm{~K}-1\right) \bar{\lambda}(M)}+\mathrm{K}}<1,
\end{array}\right.
\end{aligned}
$$

The additional condition imposed on the direction of the potential field, ensures that the transition to no-contact mode will not be followed immediately by a transition to contact mode. Thus the set of conditions (33) not only ensure stability but decrease chattering.

Gausian noise of variance $\sigma^{2}$ can be taken into account in control design, by creating a "deadzone region" around $\mathrm{f}=0$ of width $\pm 3 \sigma$, which corresponds to a confidence interval for $\mathrm{f}=0$ at a level approximately equal to $99 \%$. Thus, the switching condition for the guard $G_{n c}$ in (33a) is modified to: $|\mathrm{f}|>3 \sigma$. In this way, transitions to contact mode will only be triggered whenever a statistically significant (nonzero) value for $\mathrm{f}$ is detected. It will be demonstrated in Section 6.1.2, that even without this measure, the closed loop system maintains its stability properties.

If the conditions of Propositions 5 and 6, were satisfied, and if the hybrid system of Figure 2, had closed loop dynamics given by (17) and (19) in the contact and no-contact mode respectively, then it would be asymptotically stable. In the following section we will address the fact that (17) only expresses the ideal dynamics in the contact mode, that is, the behavior of the system if the task space coordinates were controlled directly. This is the case for a mobile robot, but not for a redundant manipulator. The proposed 
redundancy resolution scheme imposes a perturbation to (17) and results in a full state space model in the contact mode which is described by (18). The subject of the next section is to show that (18) converges very quickly to (17) (in a singular perturbations sense) and therefore the stability of the closed loop hybrid system can be established.

\subsection{Closed Loop Hybrid Dynamics}

Based on Definition 1 we are now ready to define the hybrid automaton shown in Figure 2:

The hybrid automaton $\mathcal{H}$ describing the closed loop dynamics is the following collection: $\mathcal{H}=\{\mathcal{Q}, \mathcal{X}, \mathcal{F}$, Init, $\mathcal{D}, \mathcal{E}, \mathcal{G}, \mathcal{R}\}$, where

- Discrete states $\mathcal{Q}=\{c, n c\}$;

- Continuous states of the form $z=\left(x, \mathrm{x}_{1}, x_{2}, \mathrm{x}_{3}\right)$ in $\mathcal{X} \subset \mathbb{R}^{6}$;

- Domain, which depending on whether the system is in contact with an obstacle in $\mathcal{B}$, is defined as $\mathcal{D}=\left\{z \in \mathbb{R}^{6} \| x \in \mathcal{B}\right\}$, if $q=c$, or $\mathcal{D}=\left\{\left(x, x_{2}\right) \in \mathbb{R}^{4} \mid x \notin \mathcal{B}\right\}$, if $q=n c$.

- Dynamics based on the closed loop equations for each mode, (18) and (19): $\mathcal{F}$ is given by (18) if $q=c$, or by (19), if $q=n c$.

- Initial set in the free space: Init $=\{(n c, z) \mid z \in \mathcal{X}\}$

- $\mathcal{E}=\mathcal{Q} \times \mathcal{Q}$ is the edges set;

- Guards $\mathcal{G}$ defined in (33); 
- and reset map setting some states to zero at each transition:

$$
\mathcal{R}= \begin{cases}\left(x, x_{2}\right) \rightarrow\left(x, \mathrm{x}_{1}, x_{2}, \mathrm{x}_{3}\right)=\left(x, 0, x_{2}, 0\right), & \text { for } e=(n c, c) \\ \left(x, \mathrm{x}_{1}, x_{2}, \mathrm{x}_{3}\right) \rightarrow\left(x, x_{2}\right), \quad \mathrm{x}_{1}=\mathrm{x}_{3}=0, & \text { for } e=(c, n c) .\end{cases}
$$

\subsection{Stability During Redundancy Resolution}

We combine the task-space and joint-space dynamics of the closed loop system (18)-(16) in a singular perturbation form, using $\epsilon=\frac{1}{K_{v j}}$ as the singular parameter. This system representation is written analytically as follows:

$$
\begin{aligned}
{\left[\begin{array}{c}
\dot{\mathrm{x}}_{1} \\
\dot{x}_{2} \\
\dot{\mathrm{x}}_{3}
\end{array}\right] } & =\left[\begin{array}{ccc}
0 & -\eta^{T} & 0 \\
\mathrm{~K}_{F} \mathrm{~K} \eta & -\mathrm{K}_{D} & \mathrm{~K}_{I} \mathrm{~K} \eta \\
1 & \mathbf{0}^{T} & 0
\end{array}\right]\left[\begin{array}{l}
\mathrm{x}_{1} \\
x_{2} \\
\mathrm{x}_{3}
\end{array}\right]+\left[\begin{array}{c}
0 \\
\frac{1}{\epsilon}\left(\dot{\theta}_{d}-\dot{\theta}\right) \\
0
\end{array}\right] \\
\epsilon \ddot{\theta} & =\epsilon \ddot{\theta}_{d}+\left(\dot{\theta}_{d}-\dot{\theta}\right)
\end{aligned}
$$

where $\dot{\theta}_{d}, \ddot{\theta}_{d}$ and $\dot{x}_{d}$ are reference signals, given (10), (13) and (15):

$$
\begin{aligned}
& \ddot{x}_{d}=-\mathrm{K}_{D} \dot{x}_{2}+\mathrm{K}\left(\mathrm{K}_{F} \mathrm{x}_{1}+\mathrm{K}_{I} \mathrm{x}_{3}\right) \\
& \ddot{\theta}_{d}=J^{\dagger}\left(\ddot{x}_{d}-\dot{j} \dot{\theta}_{d}\right) \\
& \dot{\theta}_{d}=J^{\dagger}\left(\dot{x}_{d}+\mathrm{v}_{d} \tau\right)+\mathrm{K}_{o}\left(I-J^{\dagger} J\right) \frac{\partial \beta}{\partial \theta}
\end{aligned}
$$

By expressing the closed loop system in the form of (34) we make the implicit assumption that the joint space dynamics (34b) are (or can be made) much faster than the task space dynamics (34a), by tunning $\mathrm{K}_{v j}$.

Proposition 7. The closed loop system (34) is exponentially stable.

Proof. The boundary layer system (34b) has an isolated equilibrium at $\dot{\theta}=$ $\dot{\theta}_{d}=J^{\dagger}\left(\dot{x}_{d}+\mathrm{v}_{d} \tau\right)+\mathrm{K}_{o}\left(I-J^{\dagger} J\right) \frac{\partial \beta}{\partial \theta}$. Substituting the steady state solution 
of (34b) in (34a) we obtain the reduced system dynamics (17), which was shown in Proposition 2 to be exponentially stable. Invoking Theorem 11.4 in $^{14}$, the combined system (34) is shown to be exponentially stable, provided that $K_{v j}>0$.

\section{Simulation Results}

We consider a point-mass mobile robot and a 3-link manipulator inside the planar workspace, similar to that of Figure 1. The task is to move from an initial configuration to a destination, without having any knowledge about the existence, shape, and location of obstacles. We present two sets of simulation results, one for each type of system. In addition to verifying the convergence properties of the proposed control scheme, we also investigate the robustness of the closed loop scheme to measurement noise, workspace complexity, navigation method singularities, and model parameter uncertainty.

Tests with varying environment stiffness have also been conducted, but are not included due to space limitations. Increased stiffness naturally produces more pronounced force transients, but stability is not affected. The interested reader is referred to ${ }^{34}$.

\subsection{Mobile Robot Simulations}

\subsubsection{Navigation and Force Regulation}

Consider an example of the navigation problem where a robot is required to move in a two-dimensional unknown workspace containing a disk and a П-shaped obstacle. Both obstacles are assumed to be of cardboard mate- 


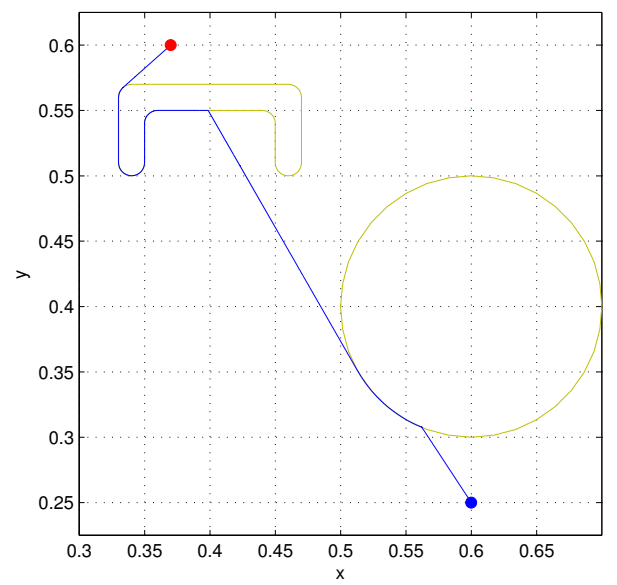

Figure 5: A mobile robot moves from $(0.6,0.25)$ to $(0.37,0.6)$, sliding along the surface of a disk and $\Pi$ shaped unknown obstacles.

rial, which has an approximate stiffness of $10^{4} \mathrm{~N} / \mathrm{m}$. Figure 5 shows the path of the mobile robot, starting at $x=(0.6,0.25)$ and converging to $x_{D}=(0.37,0.6)$. The navigation function used in this example is a quadratic function of the Euclidean distance from $x_{D}$, since no prior knowledge about obstacles in the workspace is assumed. The robot deviates from the straightline path to goal to follow the obstacle boundaries. Its velocity variation along this path is shown in Figure 6. Figures 6-7 show the evolution of the mobile robot's state during the period of 100 simulation seconds after initialization: During a small time interval within the first 10 seconds of the motion, the robot encounters the disk-shaped obstacle. Contact-mode motion is indicated by gray background in Figure 7 . At that time, the velocity of the robot drops abruptly due to collision, as shown in in Figure 6, and stabilized to a reference speed $\mathrm{v}_{d}$, set to a percentage of the speed at impact, 


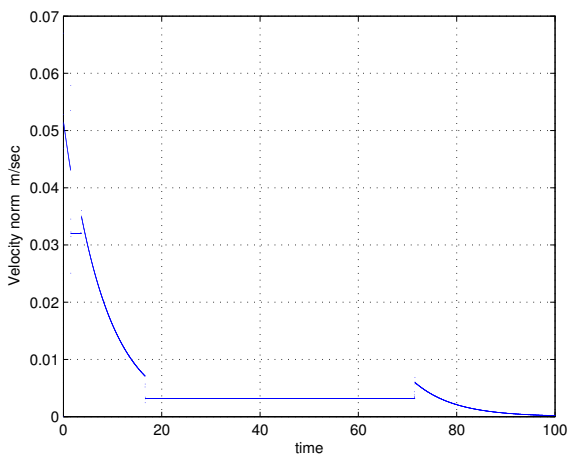

Figure 6: The 1-norm of the mobile robot's velocity vector. Regions of "depression" are associated with reduced velocity after collision with obstacles.

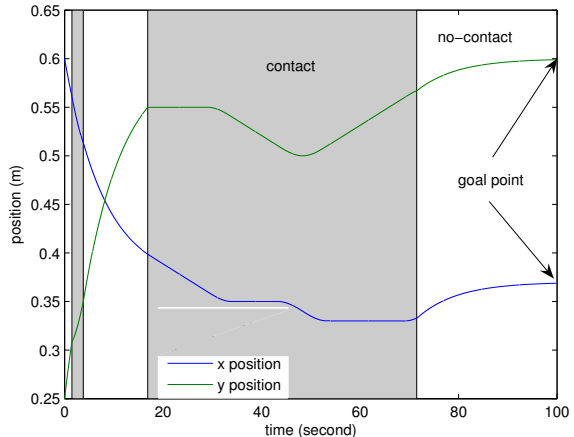

Figure 7: Evolution of the mobile robot's $x$ and $y$ coordinates, from initial point to destination. Shaded regions indicate sliding along an obstacle's boundary.

along the obstacle surface. When the switching conditions (33b) of the guard $\mathcal{G}_{c}$ are satisfied, the robot's velocity quickly converges to the potential field at the exit point, after which it continues to decrease due to the action of the damping term in (6). In the case of the mobile robot, where the inertia matrix $M$ is a constant scalar, the switching conditions (33b) are significantly simplified to the point that we can easily track the transition by observing the magnitudes of the entry and exit velocity in the contact mode. The second column of Table 1 indicates how the guard (33b) evaluates true at the exit point on disk obstacle surface. We see that the value of the navigation function is smaller at the exit point $x_{e}$, that the exit velocity $\left\|x_{2_{e}}\right\|$ is smaller than the velocity when the robot first hit the obstacle, and that the potential field direction points toward the exterior of the obstacle, along $-\eta$. 


\begin{tabular}{|c|c|c|}
\hline Switching Criteria & Disk obstacle & $\Pi$ obstacle \\
\hline$\phi\left(x_{h}\right)$ & 0.9692 & 0.0266 \\
\hline$\phi\left(x_{e}\right)$ & 0.6617 & 0.0192 \\
\hline$\left\|x_{2_{h}}\right\|$ & 0.0314 & 0.0071 \\
\hline$\left\|x_{2_{e}}\right\|$ & 0.0278 & 0.0034 \\
\hline$-\nabla \phi\left(x_{e}\right)^{T} \eta$ & $-1.0534 \times 10^{-5}$ & $-5.008 \times 10^{-5}$ \\
\hline
\end{tabular}

Table 1: Satisfying the conditions of $\mathcal{G}_{c}$, in the scenario of Figure 5.

\subsubsection{The Effect of Measurement Noise}

In the preceding example, force regulation was performed under the assumption that force measurements reflected the exerted forces accurately. However, force measurements are notoriously noisy. To simulate a realistic sensor model, force measurements are contaminated with Gaussian noise.

Noise is assumed a random signal, following a normal distribution with zero mean and a certain variance $\sigma^{2}$. We have adopted a value for the standard deviation $\sigma$ based on the experimental data collected and analyzed by Reference ${ }^{37}$. The values borrowed from ${ }^{37}$ turned out to be too small to affect our control laws, so we conservatively adjusted them by a level of magnitude and used $\sigma=0.001 \mathrm{~N}$ for our first simulation. In a subsequent simulation test, we increased $\sigma$ to $0.005 \mathrm{~N}$.

Figure 8 shows the state response of the mobile robot system when force measurements are contaminated by noise with standard deviation equal to $\sigma=0.001 \mathrm{~N}$. While moving in free space, the controller switches rapidly between contact and no-contact mode, perceiving measurement noise as a nonzero value for $\mathrm{f}$. This fast switching impacts the velocity dynamics, be- 
cause in the two modes, velocity is being controlled differently. In Figure 8 this effect is shown as chattering for the duration of the collision free motion. The guard conditions for switching back to no-contact mode are satisfied throughout this interval, allowing the rapid switching between modes. Due to the limited time spend in "contact mode" and the erroneous interpreta-

tion of the noise signal, the contact mode state variable $\mathrm{x}_{3}=\int_{t 1}^{t 2} \mathrm{x}_{1} \mathrm{~d} s$ cannot be decreased. Once true contact is made, however, approximately after 20 simulation seconds, the conditions of $\mathcal{G}_{c}$ are no longer satisfied. This forces the system to remain in contact mode, allowing the $\mathrm{x}_{3}$ state to decrease. As velocity decreases with time because of damping, chattering during free motion subsides (in terms of velocity magnitude); this is why at the later stages of motion, close to destination, it is hardly observable in Figure 8.

The overall effect of unmodelled measurement noise is performance degradation and slower speed. It can be observed in Figure 8 that the robot spends more time in contact with obstacles and convergence to destination is decelerated, without however a significant effect on the system's stability.

\subsubsection{Navigation in Cluttered Environments}

Workspace complexity does not affect the stability properties of the hybrid control law. This is demonstrated in the following simulation test, where the disk-shaped obstacle is replaced by a maze-shaped obstacle. Figure 9 shows the path of the mobile robot, starting from a configuration inside the maze, and converging to the destination point at coordinates $(0.4,0.6)$. The robot follows the contour of the maze, switching between contact and no-contact mode several times. In Table 2 there are three different exit points at the 


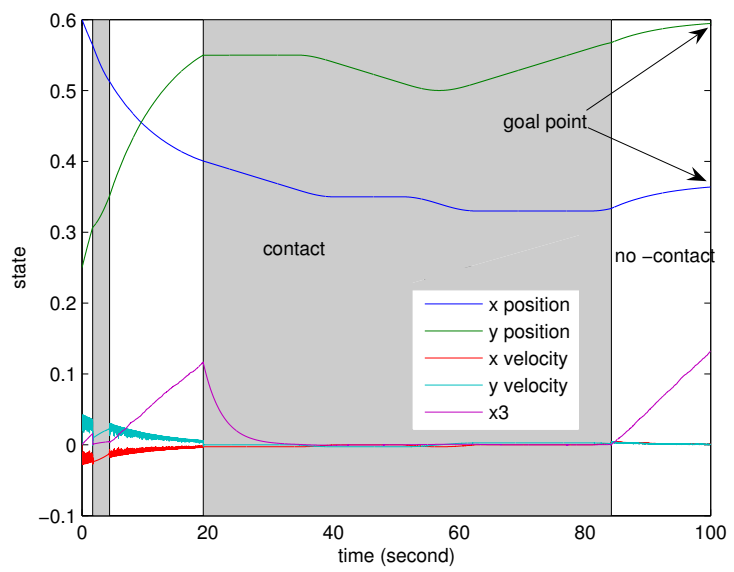

Figure 8: State response of the mobile robot in the case where force measurements are contaminated with noise of $\sigma=0.001 \mathrm{~N}$. Chattering during free motion indicates rapid switching between contact and no contact mode. The system generally decelerates, and assuming collision almost constantly, maintains an $\mathrm{x}_{3}$ state that cannot be decreased. 


\begin{tabular}{|c|c|c|c|c|}
\hline Criteria & \multicolumn{3}{|c|}{ Maze obstacle } & П obstacle \\
\hline$\phi\left(x_{h}\right)$ & 0.3554 & 0.2526 & 0.2098 & 0.0202 \\
\hline$\phi\left(x_{e}\right)$ & 0.3175 & 0.2519 & 0.1647 & 0.0201 \\
\hline$\left\|x_{2_{h}}\right\|$ & 0.0251 & 0.0232 & 0.0193 & 0.0060 \\
\hline$\left\|x_{2_{e}}\right\|$ & 0.0142 & 0.0121 & 0.0166 & 0.0059 \\
\hline$-\nabla \phi\left(x_{e}\right)^{T} \eta$ & $-5.283 \times 10^{-4}$ & -0.0051 & $-3.255 \times 10^{-4}$ & -0.0024 \\
\hline
\end{tabular}

Table 2: Evaluations indicating the satisfaction of the switching conditions for $G_{c}$ in the scenario depicted in Figure 9.

surface of the maze obstacle, and the value of $\phi\left(x_{e}\right)$ at each point suggests that the robot is "closer" (as measured by the potential function) to the goal than it was at the time when the last collision occurred.

Figure 10 shows the evolution of the contact force during contact, demonstrating that the contact-mode control law is successful in regulating the contact force to a desired level of $0.1 \mathrm{~N}$. Spikes on the force graph correspond to collision events.

\subsubsection{Potential Field Singularities}

The direction along which the robot slides on the obstacle boundary depends on the how the vector normal to the obstacle surface, $\eta$, and the potential field direction $-\nabla \phi$ align at the point of contact. This is measured by their inner product, $-\eta^{T} \nabla \phi$, and in theory when this product vanishes (the potential field direction being normal to the obstacle surface at $x_{h}$ ) there is no way to determine a direction of motion. In practice, however, this is rarely the case.

The reason is that numerical errors (or noise during experimental imple- 


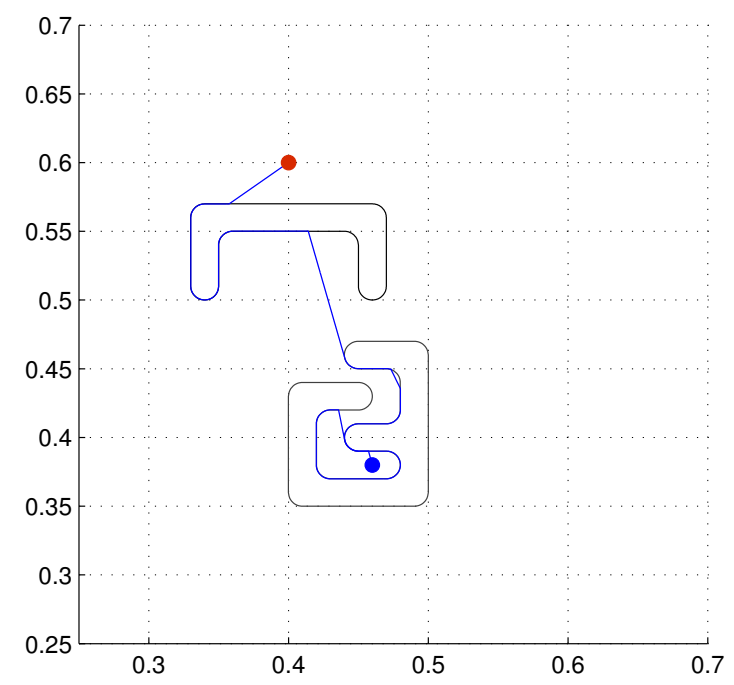

Figure 9: Mobile robot navigation in a workspace with a maze of unknown shape. The $x$ and $y$ coordinates are measured on the horizontal and vertical axes, respectively. The goal configuration has coordinates $(0.4,0.6)$.

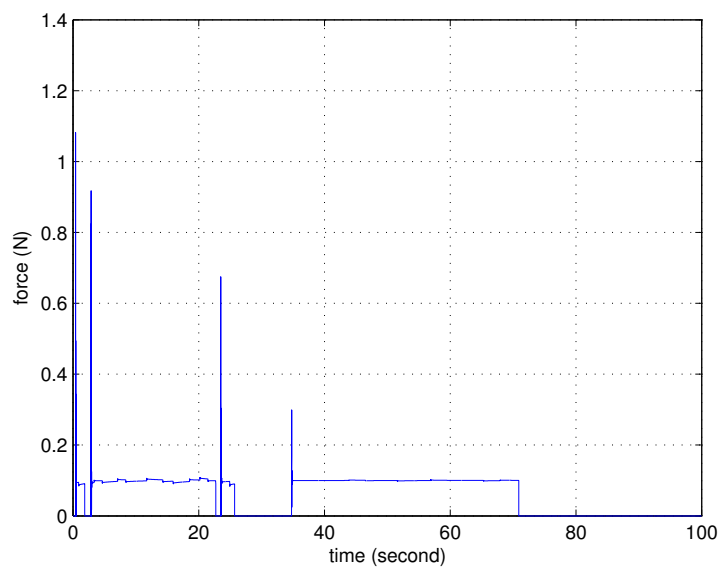

Figure 10: The contact force is regulated to $0.1 \mathrm{~N}$ during the contact phases in the simulation scenario described in Figure 9. 


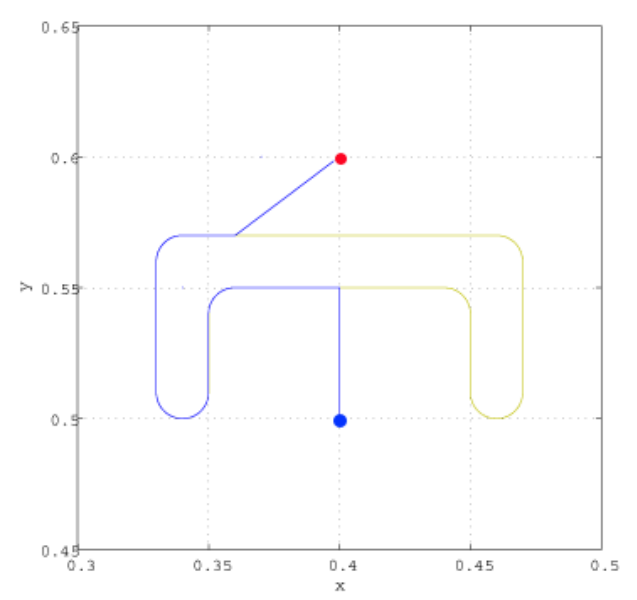

Figure 11: Despite hitting the obstacle along a direction normal to the surface, small numerical errors are sufficient to produce a tangential motion direction along the boundary.

mentation) typically provide a direction for motion. What is important to note is that the direction itself is immaterial: it is the switching conditions in $G_{c}$ that ensure the system's convergence. The robot can follow different paths to find an appropriate exit point $x_{e}$ on the obstacle's surface. We demonstrate this fact in the simulation test shown in Figure 11. Despite hitting the obstacle orthogonally, a small tangential component (in the order of $10^{-6}$ ) is sufficient to trigger the contour following behavior.

\subsection{Redundant Manipulator Simulations}

In these scenarios, a three-link manipulator moves in the two-dimensional workspace of Figure 12. The figure depicts the manipulator at an arbitrary position, amidst a disk-shaped and a $\Pi$ shaped obstacle, and with the ini- 
tial and goal configurations marked by points at locations $(0.6,0.25)$ and $(0.375,0.6)$, respectively. Circles along the manipulator body indicate the position of the rotational joints, and control points for collision avoidance have been defined along the manipulator links at regular intervals of $0.025 \mathrm{~m}$. The lengths of link 1, 2, and 3 are $0.4 \mathrm{~m}, 0.3 \mathrm{~m}$ and $0.1 \mathrm{~m}$, respectively.

Figure 13 is a superposition of snapshots showing the manipulator configuration at different time instances. The path of the manipulator, from initial configuration to goal, is also marked. As shown in an intermediate snapshot in Figure 13, the use of control points cannot eliminate the possibility of unexpected collision between the links of the robot and the obstacles completely: the tip of link 3 is shown to have "entered" the interior of the $\Pi$ shaped obstacle, toward its right "leg." However, the collision avoidance control component is indeed active and manifests itself in Figure 14 which shows the evolution of the manipulator's joint angles. In Figure 14 we see a fast change in joint angle $\theta_{3}$ at approximately $t=2 \mathrm{~s}, t=32 \mathrm{~s}$, and $t=45 \mathrm{~s}$.

The stability of joint velocities is demonstrated in Figure 16, which also shows a (bounded) velocity increase during motion in free space. The end effector force during the contact mode operation is regulated at the reference value of $0.1 \mathrm{~N}$ as shown in Figure 15.

\subsubsection{Sensitivity to Model Parameter Variations}

One of the main concerns when performing computed-torque type compensation is the fact that a robot model is rarely perfect, and consequently terms do not cancel completely as they are supposed to in theory. In this section we investigate the sensitivity of the closed loop system to uncertainty 

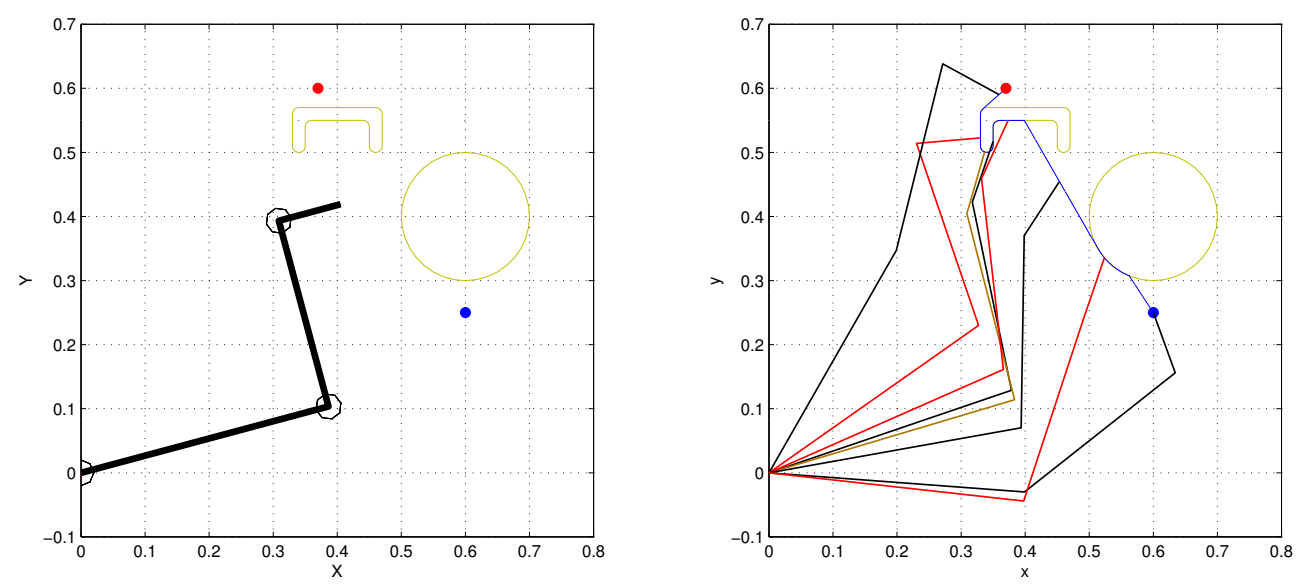

Figure 12: The 3-link manipulator Figure 13: Snapshots of different considered, at a random configuration manipulator configurations along its in a workspace with a disk and a $\Pi$ path from initial to goal position. shaped unknown obstacles.
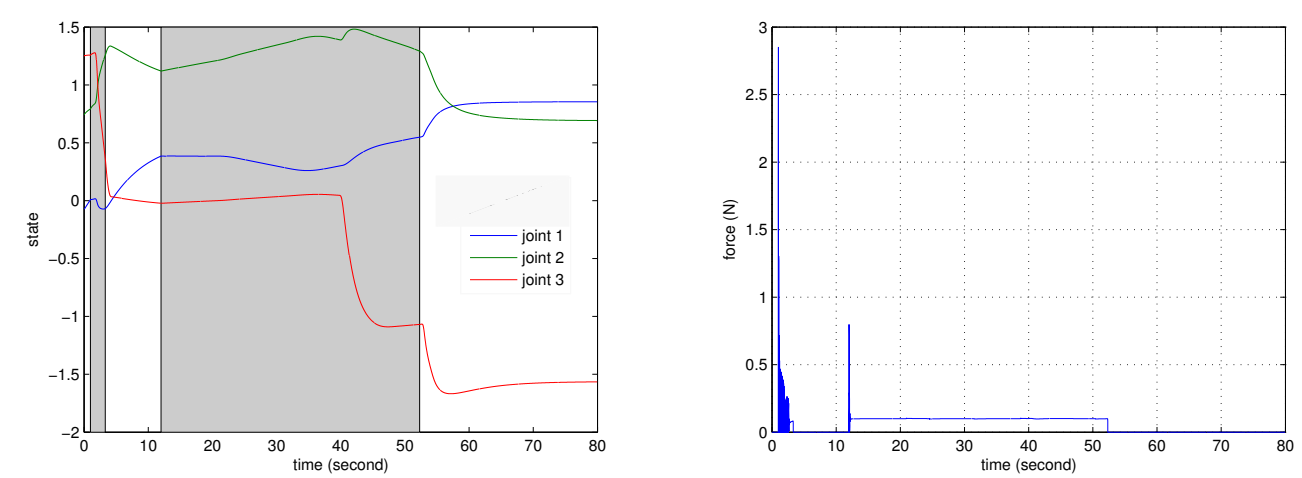

Figure 14: Joint angle trajectories. Figure 15: During the contact phase, Shaded regions correspond to time in- the exerted force is regulated at $0.1 \mathrm{~N}$. tervals when the end effector is in contact with obstacles. 


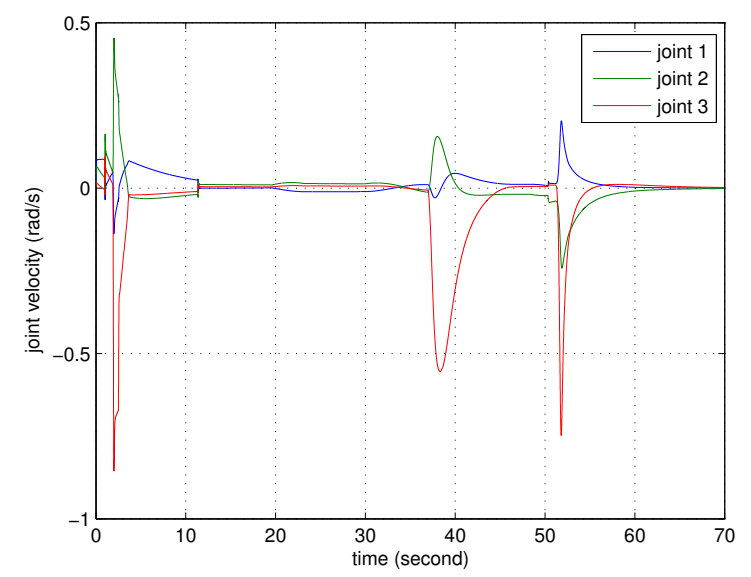

Figure 16: Trajectories of the joint angle rates. Collision avoidance maneuvers are performed for the control points, resulting in local and temporal increase of joint velocities.

in the parameters of the manipulator dynamics. We do so by artificially introducing errors in terms of link length and mass and we monitor how the performance of the system is affected. Table 3 lists the nominal values for the model parameter, along with the adjusted parameter values used for control implementation during our simulation tests.

Figures 17, 18 and 19 show the state response of the system when the controller is implemented using parameter values which are 10\%, 20\% and $50 \%$ off their nominal values, respectively. There are no significant differences in the trajectory profiles, apart from minor changes during transient phases. We attribute the seeming immunity of the system to such parameter variations to the action of the integral control during force regulation, and the use of potential fields for navigation. 


\begin{tabular}{|c|c|c|c|c|c|c|c|c|}
\hline & Length & $(\mathrm{m})$ & & & Mass & $(\mathrm{kg})$ & & \\
\hline & $\begin{array}{c}\text { Nominal } \\
\text { value }\end{array}$ & $\begin{array}{c}10 \% \\
\text { error }\end{array}$ & $\begin{array}{c}20 \% \\
\text { error }\end{array}$ & $\begin{array}{c}50 \% \\
\text { error }\end{array}$ & $\begin{array}{c}\text { Nominal } \\
\text { value }\end{array}$ & $\begin{array}{c}10 \% \\
\text { error }\end{array}$ & $\begin{array}{c}20 \% \\
\text { error }\end{array}$ & $\begin{array}{c}50 \% \\
\text { error }\end{array}$ \\
\hline Link 1 & 0.4 & 0.44 & 0.48 & 0.6 & 1 & 1.1 & 1.2 & 1.5 \\
\hline Link 2 & 0.3 & 0.33 & 0.36 & 0.45 & 1 & 1.1 & 1.2 & 1.5 \\
\hline Link 3 & 0.1 & 0.11 & 0.12 & 0.15 & 1 & 1.1 & 1.2 & 1.5 \\
\hline
\end{tabular}

Table 3: Dynamic model parameters for the 3-link manipulator. Nominal values are used to implement the manipulator model during simulation. Modified values (with errors) are used for controller implementation.

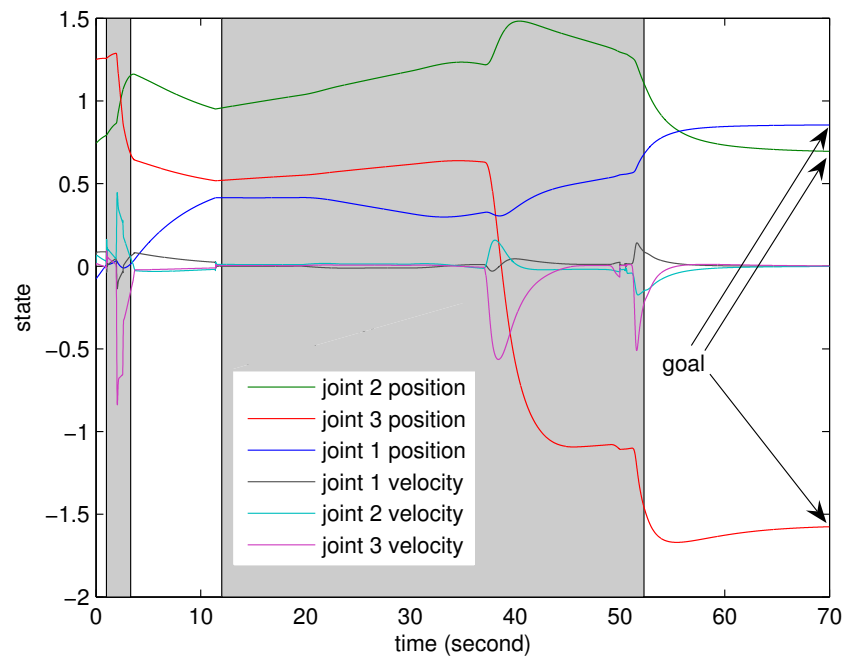

Figure 17: State Response with $10 \%$ error in the dynamic model parameters 


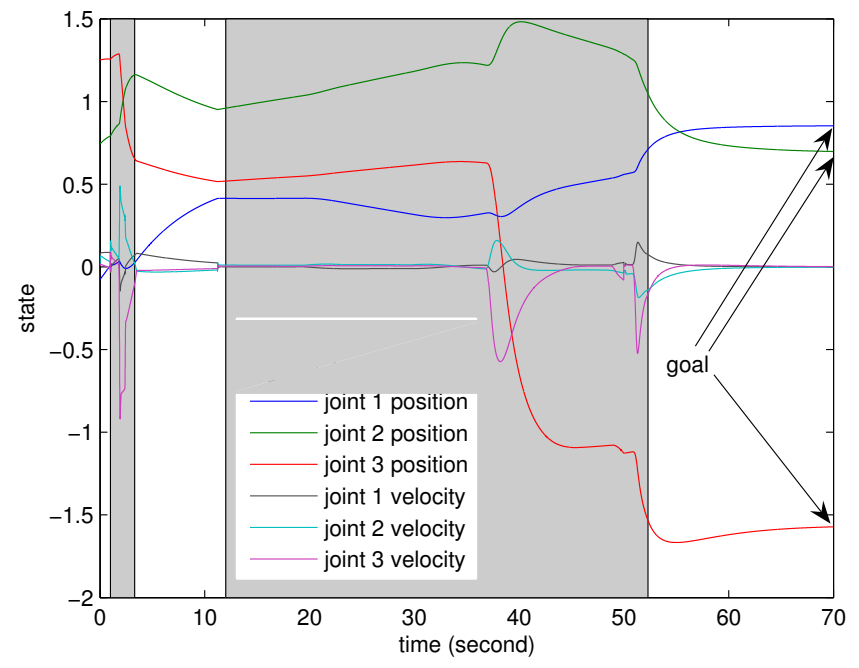

Figure 18: State Response with $20 \%$ error in the dynamic model parameters

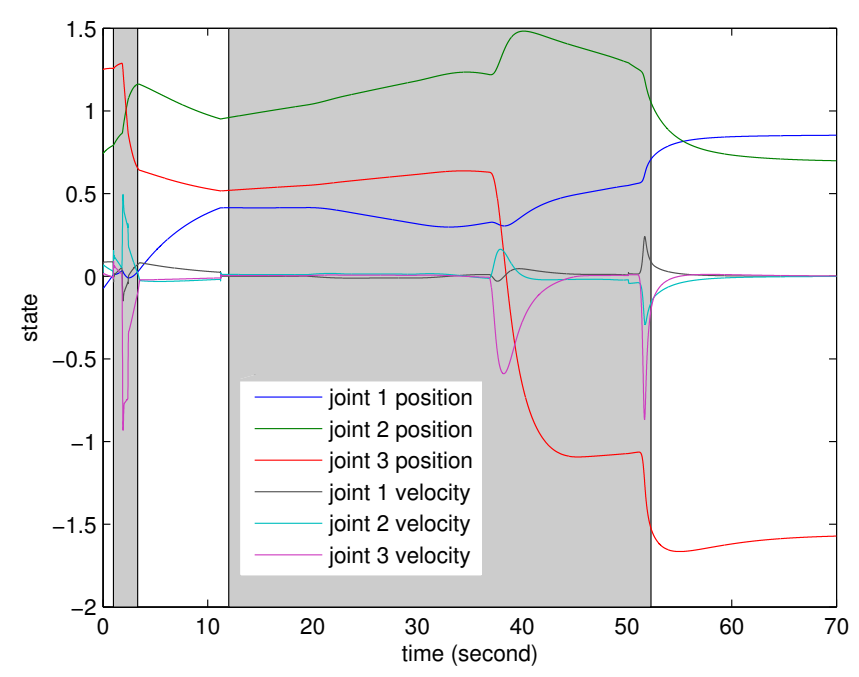

Figure 19: State Response with $50 \%$ error in the dynamic model parameters 


\section{Concluding Remarks}

We presented a hybrid control scheme for mobile robots and redundant manipulators equipped with force sensors, which allows them to navigate in an unknown environment and recover from unexpected collisions. The control law consists of two discrete modes, a non-contact mode, for motion in the free space, and a contact mode, for sliding along the surface of obstacles discovered after collision. Switching conditions between the two modes are designed to ensure the stability of the closed loop system. The contact mode control law features a velocity control action and a PI force control action. The non-contact mode combines path-planning and control action into a single control law by means of artificial potential fields. Force measurements are utilized for force regulation, obstacle detection and switching decisions. We exploited the kinematic redundancy of the manipulator to avoid collisions with obstacles discovered during execution.

The stability of the force/potential field control scheme is analyzed in a multiple Lyapunov function framework, under the assumption of a compliant environment. Switching conditions from the contact to no-contact mode and selection criteria for feedback gains are established to prove asymptotic stability for the system. Numerical simulations demonstrate the convergence properties of the closed loop system. The latter is also tested against measurement noise and model parameter variations, and results reveal some robustness, which could be a result of integral control action for force regulation and potential field for motion planning. Further work is needed to determine the source and the extend of these properties. These preliminary simulation studies are promising, and seem to encourage further experimental testing. 


\section{References}

1. V. Feliu A. Garcia and J.A. Somolinos. Experimental testing of a gauge based collision detection mechanism for a new three-degree-of-freedom flexible robot. Journal of Robotic Systems, 20:271-284, 2003.

2. Ronald C. Arkin and Robin R. Murphy. Autonomous navigation in a manufacturing environment. IEEE Transactions on Robotics and Automation, 6:445-454, 1990.

3. Michael S. Branicky. Studies in Hybrid Systems: Modeling, Analysis, and Control. PhD thesis, Massachusetts Institute of Technology, 1995.

4. Michael S. Branicky. Multiple lyapunov functions and other analysis tools for switched and hybrid systems. IEEE Transactions on Automatic Control, 43(4):475-482, 1998.

5. R.A. Brooks. A robust layered control system for a mobile robot. IEEE Journal of Robotics and Automation, RA-2:14-23, 1986.

6. S. Chiaverini and L. Sciavicco. The parallel approach to force/position control of robotic manipulators. IEEE Transactions on Robotics and Automation, 9:361-373, 1993.

7. S. Chiaverini and B. Siciliano. On the stability of a force/position control scheme for robot manipulators. In Proceedings of IFAC Robot Control, pages 183-188, 1991.

8. J. Duffy. The fallacy of modern hybrid control theory that is based on 
'orthogonal complements' of twist and wrench spaces. Journal of Robotic Systems, 7:139-144, 1990.

9. D. M. Ebert and D.D. Henrich. Safe human-robot-cooperation: Image based collision detection for industrial robots. In Proceedings of 2002 IEEE International Conference on Intelligent Robots and Systems, pages 1826-1831, 2002.

10. Q. Wei H. Zhang, Z. Zhen and W. Chang. The position/force control with self-adjusting select-matrix for robot manipulators. In Proceedings of 2001 IEEE International Conference on Robotics and Automation, pages 3932-3936, 2001.

11. J. Hollerbach and K. Suh. Redundancy resolution of manipulators through torque optimization. IEEE Journal of Robotics and Automation, RA-3:308-316, 1987.

12. Mikael Johansson and Anders Rantzer. Computation of piecewise quadratic Lyapunov functions. In Proceedings of IEEE Conference on Decision and Control, pages 3515-3520, 1997.

13. Mikael Johansson and Anders Rantzer. Computation of piecewise quadratic Lyapunov functions for hybrid systems. IEEE Transactions on Automatic Control, 43:555-559, 1998.

14. Hassan Khalil. Nonlinear Systems. Pearson Education, 2000.

15. O. Khatib. A unified approach for motion and force control of robot manipulators: The operational space formulations. IEEE Journal of Robotics and Automation, 3:43-53, 1987. 
16. Oussama Khatib. Real-time obstacle avoidance for manipulators and mobile robots. The International Journal of Robotics Research, 5(1): 90-98, 1986.

17. Amit Kumar and Herbert Tanner. Formation stabilization and collision avoidance of multiple agents using navigation functions. IEEE Transactions on Robotics, 2005. submitted.

18. J.C. Latombe. Robot Motion Planning. Kluwer Academic Publishers, Boston, MA, 1991.

19. W. Li. Fuzzy logic based perception-action behavior control of a mobile robot in uncertain environments. In Proceedings of IEEE World Congress on Computation Intelligence, pages 1626-1631, 1994.

20. W. Li. Fuzzy logic based robot navigation in uncertain environments by multisensor integration. In Proceedings of 1994 IEEE International Conference on Multisensor Fusion and Integration for Intelligent Systems, pages 439-446, 1994.

21. W. Li. Perception-action behavior control of a mobile robot in uncertain environments using fuzzy logic. In Proceedings of 1994 IEEE International Conference on Intelligent Robots and Systems, pages 439-446, 1994.

22. W. Li. A hybrid nuero-fuzzy system for sensor based robot navigation in uncertain environments. In Proceedings of 1994 American Control Conference, pages 2749-2753, 1995. 
23. W. Li and K.Z. He. Sensor-based robot navigation in uncertain environments using fuzzy logic. In Proceedings of 1994 ASME International Computers in Engineering Conference, pages 813-818, 1994.

24. Daniel Liberzon. Switching in Systems and Control. Birkhäuser, 2003.

25. Daniel Liberzon and A. Stephen Morse. Basic problems in stability and design of switched systems. IEEE Control Systems Magazine, 19(5): 59-70, 1999.

26. Savvas G. Loizou, Herbert G. Tanner, Vijay Kumar, and Kostas Kyriakopoulos. Closed loop motion planning and control for mobile robots in uncertain environment. In 42th IEEE Conference on Decision and Control, volume 18, pages 2010-2015, Maui Hawaii, 2003.

27. Alessandro De Luca and Raffaella Mattone. Sensorless robot collision detection and hybrid force/motion control. In Proceedings of 2005 IEEE International Conference on Robotics and Automation, pages 1011-1016, 2005.

28. V. Lumelsky and E. Cheung. Real-time collision avoidance in teleoperated whole-sensitive robot arm manioulator. IEEE Transactions on System, Man, Cybernetics, 23:194-203, 1993.

29. Vladimir J. Lumelsky and Alexander A. Stepanov. Path-planning strategies for a point mobile automaton moving amidst unknown obstacles of arbitrary shape. Algorithmica, 2:403-430, 1987.

30. John Lygeros. Lecture notes on hybrid systems, February 2004. Notes for an ENSIETA short course. 
31. M. T. Mason. Compliance and force control for computer controlled manipulators. IEEE Transactions on System, Man, Cybernetics, 6:418$432,1981$.

32. Y. Nakamura. Advanced Robotics: Redundancy and Optimization. Addition-Wesley, 1991.

33. Kevin A. O'Neil. Divergence of linear acceleration-based redundancy resolution schemes. IEEE Transactions on Robotics and Automation, 18:625-631, 2002.

34. Dushyant Palejiya. Force/potential field controller for robot navigation in compliant unknown environment. Master's thesis, The University of New Mexico, 2005.

35. M. H. Raibert and J. J. Craig. Hybrid position/force control of manipulator. ASME Journal of Dynamic Systems, Measurement and Control, 103:126-133, 1981.

36. Elon Rimon and Daniel E. Koditschek. Exact robot navigtion using artificial potential functions. IEEE Transactions on Robotics and Automation, 8(5):501-518, 1992.

37. J. Roy and L.Whitcomb. Adaptive force control of position/velocity controlled robots: Theory and experiment. IEEE Transactions on Robotics and Automation, 18:121-137, 2002.

38. B.Siciliano S. Chiaverini and L.Sciavicco. Force/position regulation of compliant robot manipulators. IEEE Transactions on Automatic Control, 39(3):647-651, 1994. 
39. T. Murakami S. Takakura and K. Ohnishi. An approach to collision detection and recovery motion in industrial robot. In Proceedings of 15th Annual Conference of IEEE Industrial Electronics Society, pages 421-426, 1989.

40. B. Sicilliano and L. Villani. Robot Force Control. Kluwer Academic Publishers, 1999.

41. M. Spong and R. Anderson. Hybrid impedance control of robotic manipulators. IEEE Journal of Robotics and Automation, 4:549-556, 1988.

42. H. G. Tanner, S. G. Loizou, and K. J. Kyriakopoulos. Nonholonomic navigation and control of multiple mobile manipulators. IEEE Transactions on Robotics and Automation, 19(1):53-64, February 2003.

43. T. Yoshikawa. Dynamic hybrid position/force control of robot manipulators - description of hand constrains and calculation of joint driving force. IEEE Journal of Robotics and Automation, 3:386-392, 1987.

44. L.A. Zadeh. Fuzzy sets. Information and Control, 8:338-353, 1965. 